\title{
Abundance differences between the neutral and the ionized gas of the dwarf galaxy IZw 36
}

\author{
V. Lebouteiller ${ }^{1}$, D. Kunth ${ }^{1}$, J. Lequeux ${ }^{2}$, A. Lecavelier des Etangs ${ }^{1}$, \\ J.-M. Désert ${ }^{1}$, G. Hébrard ${ }^{1}$, and A. Vidal-Madjar ${ }^{1}$
}

\author{
${ }^{1}$ Institut d'Astrophysique de Paris, CNRS, 98bis boulevard Arago, 75014 Paris, France \\ ${ }^{2}$ LERMA - Bâtiment A, Observatoire de Paris, 61 avenue de l'Observatoire, 75014 Paris, France
}

Received 2 June 2003 / Accepted 17 October 2003

\begin{abstract}
We present a FUSE spectroscopic study of the nearby gas-rich, metal-deficient blue compact dwarf (BCD) galaxy IZw 36. Atomic hydrogen and many metal lines are observed in absorption against the stellar continuum of young, massive stars embedded in the ionized region. Profile fitting of absorption lines allowed us to determine abundances and investigate the chemical composition of the neutral gas. This study presents strong evidence that nitrogen is $16_{-8}^{+7}$ times less abundant in the neutral gas than in the ionized gas (all uncertainties are $2 \sigma$ ). Similarly, the oxygen abundance estimated using phosphorus as a tracer is lower in the neutral gas by a factor of $8_{-5}^{+17}$. We also find that argon is underabundant by a factor of $32_{-7}^{+8}$ and that $\log (\mathrm{Ar} \mathrm{I} / \mathrm{OI})<-3.0$ which is inconsistent with the $\mathrm{Ar} / \mathrm{O}$ ratio $-2.1 \pm 0.1$ (Lodders 2003), implying that argon is likely ionized into Ar II in the neutral medium.
\end{abstract}

Key words. galaxies: abundances - galaxies: dwarf - galaxies: individual: IZw 36 - galaxies: ISM - galaxies: starburst ultraviolet: galaxies

\section{Introduction}

Blue compact dwarfs (BCDs) are gas-rich and relatively unevolved objects, as suggested by their low metallicity (between $\sim Z_{\odot} / 50$ and $\sim Z_{\odot} / 3$ ). Their star formation history is thought to be dominated by short, intense bursts separated by long quiescent periods (Searle \& Sargent 1972). The present starburst episode gives an opportunity to compare the chemical abundances of the $\mathrm{H}$ II regions of stars recently formed with those of their surrounding extended neutral gas. One of the issues is to know whether metal abundances as measured from $\mathrm{H}$ II regions reflect the true metallicity of star-forming galaxies and whether the neutral phase is less chemically evolved than the ionized phase. Such an attempt has been made in the past using Hubble Space Telescope (HST), with inconclusive results because of the limited wavelength coverage and the difficulties in analyzing the saturated $\lambda 1302.2 \AA$ O I line (Kunth et al. 1994; Pettini \& Lipman 1995). The Far Ultraviolet Spectroscopic Explorer (FUSE, Moos et al. 2000) gives access to many transitions of critical species such as H I, N I, O I, Si II, P II, Ar I, or Fe II, allowing one to revisit the chemical composition in neutral regions and the metal enrichment processes in nearby galaxies.

The fate of the metals newly produced by starbursts in BCDs is not yet settled. Kunth \& Sargent (1986) have presented the hypothesis that the H II region of IZw 18 has enriched itself over the timescale of the burst (i.e. few $10^{6} \mathrm{yr}$ ). The $\mathrm{H}$ II region

Send offprint requests to: V. Lebouteiller, e-mail: leboutei@iap.fr could be self-polluted, resulting in higher abundances than the surrounding neutral medium. Another possibility is that, once released by massive stars, metals remain in a hot phase, unobservable immediately in optical and UV emission lines. They would then cool into molecular droplets which later on settle onto the disk (Tenorio-Tagle 1996). Mixing with the interstellar medium (ISM) has to await photodissociation of the droplets by the UV radiation of another generation of stars before their material changes the interstellar medium composition.

The study of various elements in both ionized and neutral gas in star-forming galaxies should help to constrain the enrichment processes and identify parameters that lead to possible abundance differences, such as depletion on dust grains, ionization state correction and unprocessed (less chemically evolved) gas in the line of sight.

In this paper we investigate the ISM of IZw 36 through absorption lines of species lying in the line of sight of the ionizing stellar complex within the BCD. IZw 36 is a metal-deficient BCD with $Z=Z_{\odot} / 14$ in the $\mathrm{H}$ II region (Viallefond \& Thuan 1983, hereafter V83). The HI distribution shows a core-halo structure. The ionizing cluster is associated with the H I core which contains half of the total H I mass (V83). The H I halo is diffuse and contains several clumps.

In order to compare the chemical composition of the neutral gas with respect to the ionized gas, we will consider the abundances of nitrogen, oxygen, silicon, argon, phosphorus and iron. The observations are described in Sect. 2 and the data analysis is explained in Sect. 3. The composition of the neutral 
gas of IZw 36 is derived in Sect. 4. In Sect. 5, we discuss the abundance differences between the ionized and the neutral gas.

\section{Observations}

IZw 36 was observed with FUSE on January 13, 2001 (observation Q2240101) with an integration time of $19350 \mathrm{~s}$ (7 sub-exposures) and on May 9, 2000 (observation P1072201) with $6290 \mathrm{~s}$ (3 sub-exposures). Because of the angular extent of the ionizing cluster, the largest aperture LWRS was chosen $\left(30^{\prime \prime} \times 30^{\prime \prime}\right)$. At a distance of $6 \mathrm{Mpc}$ (Table 1), 1" corresponds to $30 \mathrm{pc}$.

Data were recorded through the two LiF channels $(\approx 1000-1200 \AA)$ and the two $\mathrm{SiC}$ channels $(\approx 900-1100 \AA)$ and processed by the pipeline Calfuse 2.2.0. The spectrum of each channel results from the co-addition of several individual sub-exposures. The typical signal-to-noise ratio $(\mathrm{S} / \mathrm{N})$ per resolution element of the co-added spectra ranges from 2 below $1000 \AA$ up to 4 above $1000 \AA$. The final spectrum shows two absorption line systems separated by approximately $1 \AA$. The first at $\approx-50 \mathrm{~km} \mathrm{~s}^{-1}$ is due to the Local ISM of the Milky Way. The second is at $270_{-6}^{+5} \mathrm{~km} \mathrm{~s}^{-1}$, which corresponds to the heliocentric velocity of IZw 36 (Table 1).

\section{Data analysis}

To analyze the data, we used the profile fitting procedure Owens developed at the Institut d'Astrophysique de Paris by Martin Lemoine and the FUSE French team. This program returns the most likely values of many free parameters such as temperatures, Doppler widths, velocities, or column densities through a minimization of the difference between the observed and computed profiles of absorption lines. Furthermore, the version we used allows changes of the background level, the continuum, and the line broadening. The continua were fitted by zero- to fourth-order polynomials, depending on the spectral region. All the coefficients of the polynomials were free. An example of fitted profiles can be seen in Fig. 1.

We considered independently several groups of species, each group being defined by its turbulent velocity, its temperature, and its heliocentric velocity. As shown in Table 2, one group defines species supposed to be mainly present in the neutral gas (see also Sect. 4.2). The two other groups contain respectively species of the ionized phase and the hot gas (giving the absorption lines of $\mathrm{O} \mathrm{VI})$. Molecular hydrogen is discussed separately in Sect. 4.1.

\subsection{The line broadening}

Taking the observed total line broadening $\sigma_{\text {tot }}$ as a free parameter, we used Owens to find its most likely value in IZw 36 spectra, supposing that $\sigma_{\text {tot }}$ is constant in the FUSE spectral range. We find $\sigma_{\text {tot }}=27 \pm 3$ pixels $\left(\approx 50 \mathrm{~km} \mathrm{~s}^{-1}\right.$ at $\left.\lambda=1000 \AA\right)$.

The total broadening of the unsaturated absorption lines has several origins:

- The instrumental line spread function. We could not use the $\mathrm{H}_{2}$ lines from IZw 36 and the Galactic component, which
Table 1. Characteristics of IZw 36.

\begin{tabular}{cc}
\hline \hline Parameter & Value \\
\hline$\alpha(2000), \delta(2000)$ & $12^{\mathrm{h}} 26^{\mathrm{m}} 16^{\mathrm{s}},+48^{\circ} 29^{\prime} 37^{\prime \prime}$ \\
$M_{\mathrm{B}}$ & -14.07 \\
$v^{a}\left(\mathrm{~km} \mathrm{~s}^{-1}\right)$ & $281 \pm 4$ \\
$D^{b}(\mathrm{Mpc})$ & $5.8<D<7.9$ \\
$Z^{c}$ & $\approx Z_{\odot} / 14$ \\
\hline
\end{tabular}

${ }^{a}$ Heliocentric radial velocity.

${ }^{b}$ Distance (Schulte-Ladbeck et al. 2001).

${ }^{c}$ Metallicity of the H II region (V83).

usually allow a good estimation of the instrumental broadening, since they are not detected in our spectra (Sect. 4.1.1). For a bright point-like source, the full width at half maximum (FWHM) is $\sigma_{\text {inst }}=11$ pixels (Hébrard et al. 2002).

- The misalignments of individual sub-exposures. The final spectrum of each detection channel is obtained by co-additions requiring wavelength shifts of individual sub-exposures. This unavoidably introduces some misalignments because of the low $\mathrm{S} / \mathrm{N}$ ratio of each exposure. We estimate that such misalignments cause an additionnal broadening of the LSF of $\sigma_{\text {add }} \approx$ 10 pixels.

- The spatial distribution of the UV-bright stars within the slit. HST/FOC images (Deharveng et al. 1994) reveal that the main concentration has an extent of $\approx 4^{\prime \prime}$, corresponding to a wavelength smearing of $\sigma_{\text {spat }} \approx 7$ pixels.

- The main source of line broadening is the velocity distribution of the absorbing clouds lying in the multiple lines of sight, whose width is a priori unknown but can be deduced for an unsaturated line by $\sigma_{\text {clouds }}=\left(\sigma_{\text {tot }}^{2}-\sigma_{\text {inst }}^{2}-\sigma_{\text {spat }}^{2}\right)^{1 / 2}=22$ pixels i.e. $40 \mathrm{~km} \mathrm{~s}^{-1}$.

The influence of the resulting total broadening $\sigma_{\text {tot }}$ on the determination of column densities is discussed in Sect. 4.1.

\subsection{The turbulent velocity}

The turbulent velocity parameter $b$ of the neutral gas is well constrained by both saturated-profile and Doppler-profile lines. From profile fitting we obtain $b=12.2_{-4.2}^{+3.4} \mathrm{~km} \mathrm{~s}^{-1}(2 \sigma \mathrm{er}-$ ror bars), corresponding to a velocity dispersion FWHM of $\sigma_{\text {neutral }}=2 \sqrt{\ln 2} b=20.3 \mathrm{~km} \mathrm{~s}^{-1}$. This result is consistent with the velocity dispersion measured from $21 \mathrm{~cm}$ radio observations $\left(\sigma_{21} \mathrm{~cm} \approx 45 \mathrm{~km} \mathrm{~s}^{-1}, \mathrm{~V} 83\right)$ and the derived velocity distribution of the absorbers (see previous section): $\sigma_{21 \mathrm{~cm}}^{2} \approx$ $\sigma_{\text {neutral }}^{2}+\sigma_{\text {clouds }}^{2}$.

Of course, a significant fraction of the gas whose turbulent velocity is measured by radio observations is behind the ionizing stars in front of which absorption lines from neutral elements originate, hence the velocity structure in our case could be different (Pettini \& Lipman 1995). The effect of the determination of the turbulent velocity on column densities is discussed in Sect. 4.1.3. 
Table 2. List of the lines detected in IZw 36. MW is for the Milky Way, $\lambda$ is the rest wavelength. Velocities of each species have been computed independently; typical errors are $\pm 10 \mathrm{~km} \mathrm{~s}^{-1}$. Species are distributed into three groups (see text). $b$ and $v$ are calculated using line profiles of all species within a group.

\begin{tabular}{|c|c|c|c|}
\hline Species & $\begin{array}{l}\text { Velocity } \\
\left(\mathrm{km} \mathrm{s}^{-1}\right)\end{array}$ & $\begin{array}{r}\lambda \\
(\AA)\end{array}$ & comment \\
\hline \multicolumn{4}{|c|}{ First group $\equiv$ neutral region of IZw $36 . b=12.2_{-4.2}^{+3.4} \mathrm{~km} \mathrm{~s}^{-1}, v=270_{-6}^{+5} \mathrm{~km} \mathrm{~s}^{-1}$} \\
\hline \multirow[t]{2}{*}{$\mathrm{HI}$} & & 1025.72 & Ly $\beta$, presence of damping wings (Fig. 1a) \\
\hline & & $937.80,930.75,926.23,923.15$ & resp. $\operatorname{Ly} \epsilon, \operatorname{Ly} \zeta, \operatorname{Ly} \eta, \operatorname{Ly} \theta$ \\
\hline $\mathrm{C}$ II & 259 & 1036.34 & strongly saturated, blended with $\mathrm{C}$ II* from $\mathrm{MW}$ and $\mathrm{O}$ VI from MW \\
\hline \multirow[t]{3}{*}{ N I } & 265 & 1134.98 & not saturated (Fig. 5) \\
\hline & & 963.99 & $\begin{array}{l}\text { not saturated, blended with } \mathrm{N} \text { I from MW } \\
\text { and P II from IZw } 36\end{array}$ \\
\hline & & $954.10,953.97,953.65,953.42$ & not saturated, blended with each other \\
\hline \multirow[t]{3}{*}{ O I } & 272 & 1039.23 & strongly saturated \\
\hline & & 976.45 & saturated, blended with C III from MW \\
\hline & & 950.89 & saturated, blended with P IV from IZw 36 \\
\hline Si II & 261 & 1020.70 & slightly saturated \\
\hline \multirow[t]{2}{*}{ P II } & 270 & 1152.82 & detected \\
\hline & & 963.80 & blended with N I from MW and N I from IZw 36 \\
\hline ArI & & 1048.22 & not detected (Fig. 3) \\
\hline \multirow[t]{3}{*}{ Fe II } & 270 & 1144.94 & not saturated (Fig. 1b) \\
\hline & & $1143.23,1142.37,1125.45$ & not saturated \\
\hline & & $1121.97,1096.88,1063.18$ & not saturated \\
\hline \multicolumn{4}{|c|}{ Second group: species with ionization potentials $>29 \mathrm{eV} . b=24.3_{-16.3}^{+15.9} \mathrm{~km} \mathrm{~s}^{-1}, v=260_{-12}^{+8} \mathrm{~km} \mathrm{~s}^{-1}$} \\
\hline C III & 261 & 977.02 & detected \\
\hline N II & 254 & 1083.99 & barely detected \\
\hline PIV & 254 & 950.66 & blended with O I from IZw 36 \\
\hline S III & 248 & 1012.50 & detected \\
\hline Fe III & 264 & 1122.53 & detected \\
\hline \multicolumn{4}{|c|}{ Third group $\equiv$ hot gas. $b=51.4_{-18.7}^{+35.9} \mathrm{~km} \mathrm{~s}^{-1}, v=235_{-16}^{+23} \mathrm{~km} \mathrm{~s}^{-1}$} \\
\hline O VI & 235 & $1037.62,1031.93$ & detected \\
\hline
\end{tabular}

\section{Composition of the neutral gas}

\subsection{Column densities}

Column densities are given in Table 3. Throughout the paper, error bars are $2 \sigma$. The multiple lines of sight to the massive stars that contribute to the UV continuum may cross clouds with different chemical composition, so that the reported column densities represent global values among all the clouds.

Errors on the column densities essentially depend on where the lines are located on the curve of growth. In particular, saturated lines can give column densities spanning 3 orders of magnitude when $b$ is not constrained by lines of other species lying in the same gas phase. Errors are computed using the $\Delta \chi^{2}$ method: $\chi^{2}$ is calculated as a function of the column density. We use the difference $\Delta \chi^{2}=\chi^{2}-\chi_{\min }^{2}$, where $\chi_{\min }^{2}$ is the best fit to derive the error bars ( $2 \sigma$ error bars correspond to $\left.\Delta \chi^{2}=4\right)$. This method is also used to evaluate error bars for $b$ and the line broadening. Figure 2 shows an example of a $\Delta \chi^{2}$ curve.

The determination of the H I column density is not affected by the line broadening $\sigma_{\text {tot }}$. However, we have tested several values of $\sigma_{\text {tot }}$ in order to test its impact on the Fe II, Si II, P II, and N I column densities. It can be seen in Table 4 that for $\sigma_{\text {tot }}$ between 12 and 27 pixels, column densities do not vary by more than a factor 2.5 in the worst case (Si II). A bad determination of the line broadening would not change the results of Sect. 5.

\subsubsection{Molecular hydrogen}

$\mathrm{No} \mathrm{H}_{2}$ lines were detected at the redshift of IZw 36 (Table 3), as for IZw 18 (Vidal-Madjar et al. 2000), Markarian 59 (Thuan et al. 2002), and SBS 0335-052 (Thuan et al. 2003). There are several possible physical explanations for this absence as pointed out by Vidal-Madjar et al. (2000): the ionizing flux of the massive stars which can be high enough to destroy $\mathrm{H}_{2}$ molecules, the scarcity of dust grains on which $\mathrm{H}_{2}$ molecules are synthetized, and a low HI volumic density. Also, Vidal-Madjar et al. (2000) argue that the molecular medium could be clumpy so that the few clumps in the lines of sight of the many blue stars in the entrance aperture would not be detected. 
Table 3. Column densities derived in IZw 36. Errors are given at $2 \sigma$, upper limits at $3 \sigma$. Upper limits for $\mathrm{H}_{2}$ are calculated using the same turbulent velocity as the neutral phase $\left(12.2 \mathrm{~km} \mathrm{~s}^{-1}\right)$. The Fe III column density is calculated from the Fe III $\lambda 1122.53$ line which could originate from both the ISM and stellar atmospheres.

\begin{tabular}{|c|c|c|c|}
\hline Species & $\log N\left(\mathrm{~cm}^{-2}\right)$ & $\log (\mathrm{X} / \mathrm{H})$ & {$[\mathrm{X} / \mathrm{H}]^{a}$} \\
\hline $\mathrm{HI}$ & $21.30_{-0.10}^{+0.09}$ & & \\
\hline C II & $18.31_{-0.19}^{+0.18}$ & & \\
\hline C III & $14.38_{-0.19}^{+0.18}$ & & \\
\hline $\mathrm{N}_{\mathrm{I}}$ & $14.42_{-0.22}^{+0.26}$ & $-6.88_{-0.31}^{+0.36}$ & $-2.71_{-0.42}^{+0.47}$ \\
\hline $\mathrm{N}$ II & $14.02_{-0.98}^{+1.15}$ & & \\
\hline O I & $16.80_{-1.01}^{+1.85}$ & $-4.50_{-1.10}^{+1.96}$ & $-1.19_{-1.15}^{+2.00}$ \\
\hline OVI & $13.97_{-0.22}^{+0.16}$ & & \\
\hline Si II & $15.34_{-0.28}^{+0.85}$ & $-5.96_{-0.37}^{+0.96}$ & $-1.50_{-0.39}^{+0.97}$ \\
\hline P II & $13.00_{-0.36}^{+0.28}$ & $-8.30_{-0.45}^{+0.36}$ & $-1.76_{-0.51}^{+0.41}$ \\
\hline S III & $14.84_{-0.15}^{+0.43}$ & & \\
\hline Ar I & $<13.30$ & $<-8.00$ & $<-2.55$ \\
\hline Fe II & $14.51_{-0.14}^{+0.21}$ & $-6.79_{-0.23}^{+0.31}$ & $-2.26_{-0.26}^{+0.34}$ \\
\hline Fe III & $14.47_{-0.14}^{+0.18}$ & & \\
\hline $\mathrm{H}_{2(J=0)}$ & $<14.41$ & & \\
\hline $\mathrm{H}_{2(J=1)}$ & $<14.61$ & & \\
\hline $\mathrm{H}_{2(J=2)}$ & $<14.32$ & & \\
\hline
\end{tabular}

${ }^{a}[X / Y]=\log (X / Y)-\log (X / Y)_{\odot}$, where $\log (X / Y)_{\odot}$ is the solar value. We use the recommended solar values of Lodders (2003).

Table 4. Effects of the total line broadening $\sigma_{\text {tot }}$ (in pixels, see Sect. 3.1) on column densities $\left(\log N\left(\mathrm{~cm}^{-2}\right)\right)$. Note that if $\sigma_{\text {tot }}$ is overestimated so are the column densities.

\begin{tabular}{lccccc}
\hline \hline & $\sigma_{\text {tot }}=12$ & 15 & 21 & 24 & 27 \\
\hline $\log N(\mathrm{~N}$ I $)$ & 14.30 & 14.30 & 14.32 & 14.36 & 14.42 \\
$\log N($ Si II $)$ & 15.08 & 15.08 & 15.15 & 15.20 & 15.34 \\
$\log N($ P II $)$ & 12.99 & 13.02 & 13.14 & 13.16 & 13.17 \\
$\log N($ Fe II $)$ & 14.20 & 14.20 & 14.26 & 14.30 & 14.49 \\
\hline
\end{tabular}

\subsection{2. $\mathrm{HI}$}

The H I column density has been calculated using all the available Lyman lines ( $\operatorname{Ly} \beta$, $\operatorname{Ly} \epsilon, \operatorname{Ly} \zeta, \operatorname{Ly} \eta$, and $\operatorname{Ly} \theta)$ but is mainly constrained by the damping wings of the $\operatorname{Ly} \beta$ line (Fig. 1a) since the other $\mathrm{H}$ I lines are strongly saturated. We used a $30 \AA$ wide window centered on $\operatorname{Ly} \beta$ to constrain the continuum by a 4-order polynomial. We find $\log N(\mathrm{H} \mathrm{I})=21.30_{-0.10}^{+0.09}$. The errors on $\log N(\mathrm{HI})$ are the smallest amongst the specie we investigate. This is due to the damping profile of the $\operatorname{Ly} \beta$ line which is observed through both $\mathrm{LiF}$ and $\mathrm{SiC}$ channels.

Our value is somewhat higher than $\log N(\mathrm{HI})=21.15$ found by V83 who used only the red damping wing of the Ly $\alpha$ line from IUE observations, but is certainly better constrained in our case because of the two damping wings of the Ly $\beta$ line and because of the better resolution and the better signal-to-noise ratio of these FUSE observations.

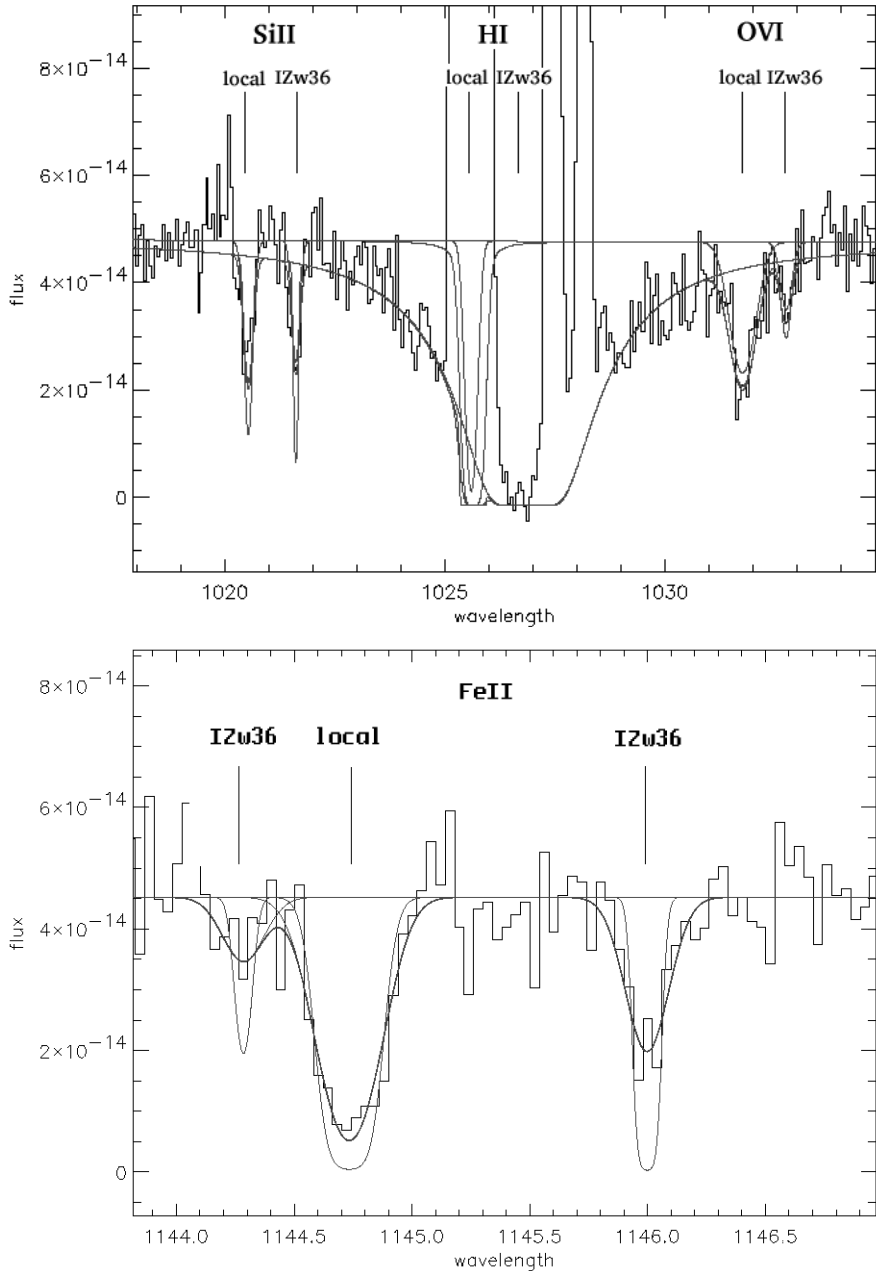

Fig. 1. H I and Fe II lines in IZw 36. The flux is in erg s $\mathrm{s}^{-1} \mathrm{~cm}^{-2} \AA^{-1}$ and the observed wavelength in $\AA$. The data (histogram) are binned by a factor 3 for display purposes. The thin line represents the line profile before the convolution with the total line broadening represented by $\sigma_{\text {tot }}$ in Sect. 3.1 and the thick line is the convolved profile. For each of the spectral windows, we used the two observations and all the available FUSE detection channels (Sect. 2) to perform the fit. Here we show the spectrum of segment LiF1 for the upper plot and of segment LiF2 for the lower plot. a) (upper) Plot of the $\operatorname{Ly} \beta$ line. The center of the line is blended with terrestrial airglow emission lines. Atomic hydrogen in the Milky Way is invisible because of its low column density $\sim 10^{19} \mathrm{~cm}^{-2}$ (estimated from Heiles 1975). The Si II $\lambda 1020.70$ lines and O VI $\lambda 1031.93$ lines from the Milky Way and IZw 36 are also visible in this spectrum. b) (lower) Plot of the strong Fe II $\lambda 1144.94$ line.

\subsubsection{NI and N II}

$\mathrm{NI}$ is the most useful species in our study since most of it must be present in the neutral ISM. Six N I lines were used (Table 2). These lines are not saturated so that the determination of $\log N(\mathrm{~N} \mathrm{I})$ is not strongly dependent on the turbulent velocity $b$ (Fig. 2). We obtain $\log N(\mathrm{~N} \mathrm{I})=14.42_{-0.22}^{+0.26}$.

The $\lambda 1083.99 \mathrm{~N}$ II line is barely detected since it is located in a spectral region which is very noisy. We are however able to calculate a column density: $\log N(\mathrm{~N}$ II $)=14.02_{-0.98}^{+1.15}$. 


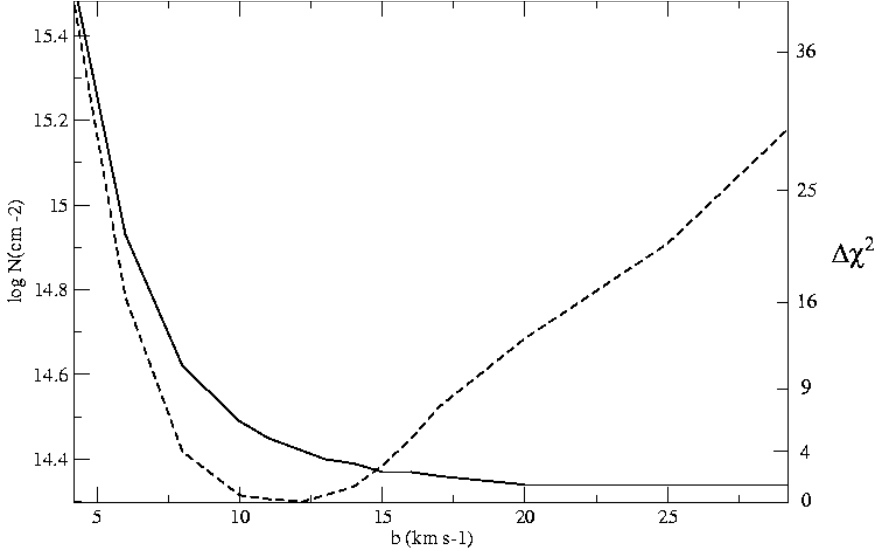

Fig. 2. Effects of the determination of the turbulent velocity $b$ on the N I column density (solid line). Errors in $b$ do not affect much the column density determination. The $\Delta \chi^{2}$ curve (dashed line) is relatively narrow and is minimal for $b=12.2 \mathrm{~km} \mathrm{~s}^{-1}$. At $2 \sigma\left(\Delta \chi^{2}=4\right)$ we obtain $b=12.2_{-4.2}^{+3.4}$ corresponding to $\log N(\mathrm{~N} \mathrm{I})=14.43_{-0.06}^{+0.19}$.

\subsection{4. $\mathrm{O}$ I and $\mathrm{P} \|$}

The three O I lines in IZw 36 are either blended or strongly saturated (Table 2), resulting in large errors (Table 3).

The $1963.80 \mathrm{P}$ II line has a large oscillator strength $(f=$ 1.46) and is detectable even for low P II column densities. In IZw 36 spectra, this line is blended with N I lines from the Milky Way and from IZw 36. Given the fact that the N I column density is well determined and that the P II line is not saturated, the blending can be corrected for. We also use the $\lambda 1152.82 \mathrm{P}$ II line with a fainter oscillator strength to constrain the final P II column density as $\log N(\mathrm{P}$ II $)=13.00_{-0.36}^{+0.28}$.

\subsubsection{Si II, Ar I, Fe ॥}

The Si II column density is only given by the $\lambda 1020.70$ line which is slighly saturated. Unfortunately, the other strong Si II line at $989.90 \AA$ is contaminated by terrestrial airglows.

The other species lead to reliable column densities: Ar I (Fig. 3) for which we have a good upper limit, and Fe II (Fig. 1b) with a large collection of lines.

\subsection{Abundances in the neutral region}

In order to derive total abundances from ionic column densities, it is necessary to know the ionization states of each species in the neutral region.

In principle, in the $\mathrm{HI}$ region we expect to find all elements with an ionization potential larger than that of hydrogen $(13.6 \mathrm{eV})$ as neutral atoms. Elements with smaller ionization potentials must be found as single-charged ions. For example, we can safely assume that iron and silicon exist mainly as Fe II and Si II in the neutral medium and that the Fe II and Si II lines are essentially produced there. We have checked that the column densities of these ions are considerably smaller in the $\mathrm{H}$ II region using the model E2E1 from the grid of Stasińska (1990). The model E2E1 agrees the best with the physical parameters of the $\mathrm{H}$ II region derived by V83. The resulting ionic

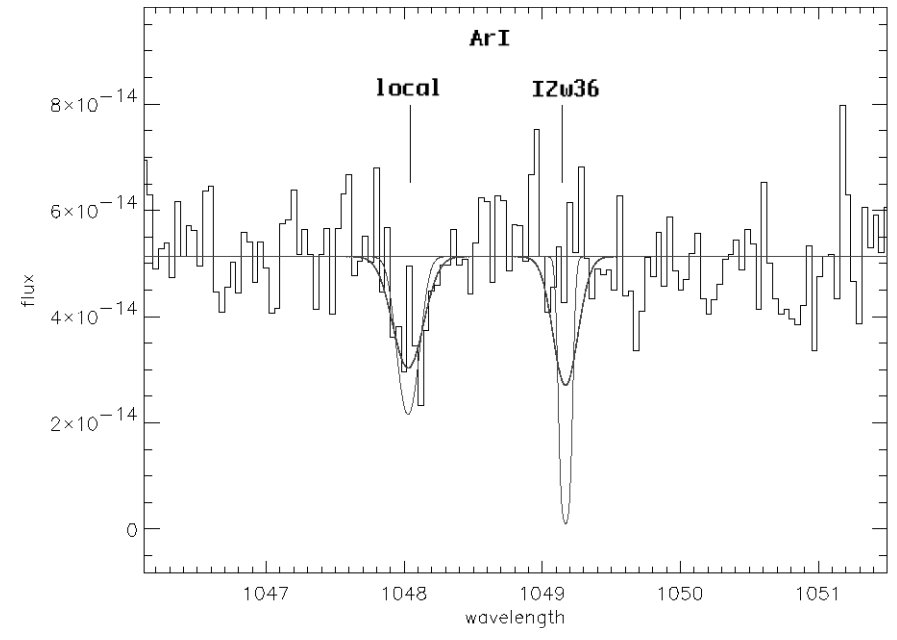

Fig. 3. Lack of argon in IZw 36. See Fig. 1 for a description of the plot. Data are binned by a factor 2 for display purposes. The line plotted at the redshift of IZw 36 is for $\log N(\operatorname{Ar} \mathrm{I})=14.0$ and $b=12.0 \mathrm{~km} \mathrm{~s}^{-1}$.

fractions of Si II and Fe II in the H II region in the best fitting model are only a few percents.

The situation is however not so simple for elements with an ionization potential not much larger than that of hydrogen, because UV photons able to produce their ionization may be present in low-density, partly-ionized regions of the ISM. For example, Ar I (ionization potential $15.8 \mathrm{eV}$ ) is easy to ionize because of its relatively large photoionization cross section (Sofia \& Jenkins 1998). Therefore, in regions with an ionization degree $n_{\mathrm{e}} / n_{\mathrm{H}} \approx 0.2$, the actual $\mathrm{Ar} / \mathrm{H}$ can be larger by 0.2 to 0.7 dex than $\mathrm{Ar} / \mathrm{HI}$, depending on the hardness of the far-UV radiation. The situation is similar but less severe for $\mathrm{O} \mathrm{I}$ and $\mathrm{N}$ I (ionization potentials $13.6 \mathrm{eV}$ and $14.5 \mathrm{eV}$ respectively). Indeed, in the conditions of the Local Cloud for which the correction for $\mathrm{Ar} \mathrm{I} / \mathrm{HI}$ is $0.36 \mathrm{dex}$, the corresponding correction for N I/HI is only 0.05 dex (Sofia \& Jenkins 1998). However the ionization correction in BCDs could be different and could reach $0.2-0.3$ dex. We will first neglect this correction, and because little N I and $\mathrm{O}$ I are expected in the $\mathrm{H}$ II region, we assume that the $\mathrm{N} \mathrm{I}$ and $\mathrm{O} \mathrm{I}$ lines are representative of the total nitrogen and oxygen abundances in the neutral region.

The abundances of the neutral region can now be compared with the ionized region values. For the comparison, we use metal abundances relative to hydrogen $(\mathrm{X} / \mathrm{H})$.

\section{Comparison with the ionized gas}

Figure 4 compares several abundances ratios representative of the ionized phase of IZw 36, derived from optical and UV emission lines, with those of the neutral gas. We define the underabundance $\delta_{\mathrm{HI}}(x)$, as the logarithmic difference of an abundance ratio $x$ between the $\mathrm{H}$ II and the H I regions by:

$\delta_{\mathrm{HI}}(x)=\log (x)_{\mathrm{HII}}-\log (x)_{\mathrm{HI}}$.

The column densities of oxygen and silicon of IZw 36 are similar in the neutral and in the ionized gas within large uncertainties. However the result for nitrogen is much more reliable, and is certainly the best established result of this study, 


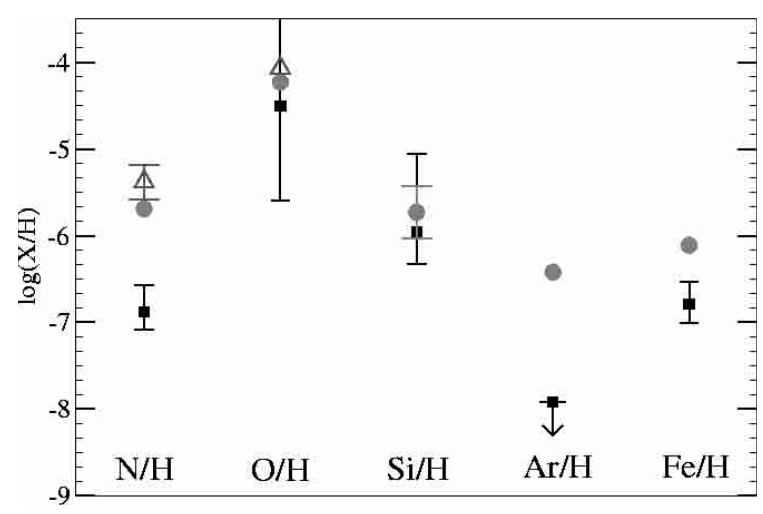

Fig. 4. Comparison of X/H between $\mathrm{HI}$ and $\mathrm{H}$ II regions in IZw 36. Error bars are at $2 \sigma$. Squares: this study (assuming the abundances of Sect. 4.2 and using Ar I for argon i.e. without any ionization correction). Triangles: V83. Circles: Izotov et al. (1997) for N, O, Ar, and Fe. The silicon abundance in the ionized region, not measured in IZw 36, is obtained by assuming $\log (\mathrm{Si} / \mathrm{O})=-1.5 \pm 0.3$ which is the mean value observed in BCDs (Izotov \& Thuan 1999). Error bars, when not mentioned, are within the points widths.

yielding $\delta_{\mathrm{HI}}(\mathrm{N} / \mathrm{H})=1.2 \pm 0.3$; in other words $\mathrm{N}$ is sixteen times less abundant in the neutral gas than in the ionized gas. This difference is larger than $3 \sigma$. Other metals for which abundances are accurately determined also show underabundances: $\delta_{\mathrm{HI}}(\mathrm{Fe} / \mathrm{H})=0.7 \pm 0.2$ and $\delta_{\mathrm{HI}}(\mathrm{Ar} / \mathrm{H})>1.5 \pm 0.1$ (without any attempt to correct for partly-ionized, low-density regions).

The following discussion aims to identify the effects responsible for the observed iron, nitrogen, argon, and oxygen underabundances.

\subsection{Iron and silicon}

A concern with the abundance of iron in $\mathrm{H}$ II regions is that it is derived from the weak $\lambda 4658$ line of Fe III, and is relatively uncertain. In any case, we find that iron is underabundant by $\delta_{\mathrm{HI}}(\mathrm{Fe} / \mathrm{H})=0.7 \pm 0.2$ in IZw 36. This underabundance might be overestimated since it is likely that iron is more efficiently depleted on dust grains in the neutral phase than in the ionized phase.

In IZw 36 , the only available Si II line is slightly saturated (Sect. 4.1.5) so that the determination of the Si II column density is uncertain. Within the large error bars, the abundances in the ionized region and in the neutral region are in agreement.

Because of all these uncertainties, and because silicon and iron can be depleted on dust grains, we prefer to focus on nitrogen abundance which is much more reliable.

\subsection{Nitrogen}

The underabundance in $\mathrm{IZw} 36 \delta_{\mathrm{HI}}(\mathrm{N} / \mathrm{H})=1.2 \pm 0.3$ is too large to be accounted for by chemical inhomogeneities alone in the neutral gas, given the underabundance of iron. In order to illustrate the underabundance of nitrogen in the neutral gas, we have compared in Fig. 5 our best fit of the $\lambda 1134.98 \mathrm{~N}$ I line, one of the 6 lines we used, with the profile assuming the

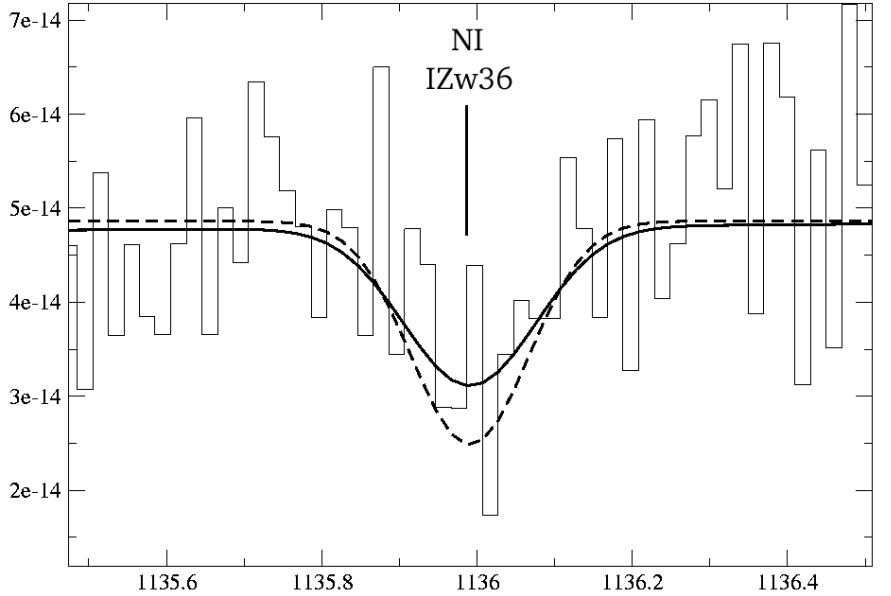

Fig. 5. Fit of the $\lambda 1134.98$ line. Data are binned by a factor 2 for display purposes. The solid line is for $\log (\mathrm{NI} / \mathrm{HI})=-6.88$ and shows our best fit. The dashed line is for $\log (\mathrm{N} \mathrm{I} / \mathrm{HI})=-5.63$ and does not fit well the data.

$\mathrm{N} / \mathrm{H}$ ratio as in the ionized gas. The two profiles are inconsistent within $3 \sigma$.

Furthermore, even including the largest possible ionization correction (i.e. including $\mathrm{N}$ II and taking the upper error bars on $\mathrm{N} \mathrm{I}$ and N II column densities), the underabundance of nitrogen is still positive: $\delta_{\mathrm{HI}}(\mathrm{N} / \mathrm{H})=1.0_{-0.9}^{+0.2}$. This supposes that $75 \%$ of the nitrogen is ionized into $\mathrm{N}$ II in the neutral gas of IZw 36 . Although the determination of N II column density is uncertain (Sect. 4.1.3), we can reasonnably conclude that nitrogen is indeed underabundant in the H I gas of IZw 36.

\subsection{Argon}

The argon deficiency is $\delta_{\mathrm{HI}}(\mathrm{Ar} / \mathrm{H})>1.5 \pm 0.1$. Argon is produced in massive stars and should not be depleted in a lowdensity H I cloud (Jenkins et al. 2000). A major part of the underabundance in the neutral gas is certainly due to an ionization correction from low-density, partly-ionized regions of the ISM (Sect. 4.2) such as argon is mainly ionized into Ar II.

\subsection{Oxygen}

The $\mathrm{O} / \mathrm{H}$ ratio in the neutral gas of IZw 36 is difficult to assess because of the large errors on the OI column density (Table 3). However, it is possible to estimate its value using other elements which are produced in the same massive stars. Phosphorus turns out to be a good tracer of oxygen (Lebouteiller 2003), since argon is partly-ionized in the neutral region (Sect. 5.3) and since the depletion of silicon on dust grains is uncertain. Depletion is not a concern for phosphorus since it is not much depleted, and in the same way as oxygen (Savage \& Sembach 1996).

We calculate $\mathrm{O}^{P}$ which estimates the neutral oxygen abundance assuming $[\mathrm{P} / \mathrm{O}] \approx 0$ in the neutral gas (using the recommended solar value of Lodders $(2003) \log (\mathrm{P} / \mathrm{O})_{\odot}=-3.23 \pm$ $0.09)$. We find $\log \left(\mathrm{O}^{P} / \mathrm{H}\right) \approx-5.1 \pm 0.5$, giving an underabundance $\delta_{\mathrm{HI}}\left(\mathrm{O}^{P} / \mathrm{H}\right) \approx 0.9 \pm 0.5$. Within the errors, this estimation of the oxygen abundance is lower than that in the ionized 
gas. Given the low depletion of phosphorus and oxygen in the Galactic ISM (André et al. 2003 and Savage \& Sembach 1996), it is very likely that oxygen is genuinely deficient in the neutral medium of IZw 36.

Assuming $\log \left(\mathrm{O}^{P} / \mathrm{H}\right)=-5.1 \pm 0.5$ in the $\mathrm{HI}$ gas, we find $\log \left(\mathrm{N} / \mathrm{O}^{P}\right)=-1.8 \pm 0.6(2 \sigma$ uncertainty) lower than, although consistent with, the value $-1.49 \pm 0.01$ in the ionized gas (Izotov \& Thuan 1999). Also, we find $\log \left(\mathrm{Ar} / \mathrm{O}^{P}\right)<$ -3.0 as compared to the solar value $\log (\mathrm{Ar} / \mathrm{O})_{\odot}=-2.1 \pm 0.1$ (Lodders 2003), confirming that argon is likely ionized into Ar II as stressed in Sect. 5.3.

\section{Conclusions}

Several heavy elements in the neutral gas of IZw 36 are underabundant in the neutral gas with respect to the ionized gas. Various effects can be responsible for the underabundances $\delta_{\mathrm{HI}}(\mathrm{X} / \mathrm{H})$ :

- The presence of an unprocessed neutral gas, less chemically evolved, in the line of sight can reduce all the abundances $\mathrm{X} / \mathrm{H}$ in the neutral gas with respect to the ionized gas.

- A more efficient depletion on dust grains in the neutral medium can account for the iron deficiency.

- An additional metal enrichment could be responsible for nitrogen and oxygen (using phosphorus as a tracer) overabundance in the ionized gas, although in this case, silicon which is an $\alpha$-element should be also overabundant (but see caption of Fig. 4).

Our findings that metals are observed in the neutral region of IZw 36 indicates that this gas phase already has been enriched by previous star formation, either quiescent or in bursts (Legrand 2000). Schulte-Ladbeck et al. (2001) detect indeed an old stellar population which could have enriched the neutral region of IZw 36.

In this study, we considered H II region abundances derived from forbidden lines in the visible and the ultraviolet. However, the abundances derived in this way might be underestimated by factors ( $~ 22$ for oxygen for instance) as claimed by Tsamis et al. (2003) compared to the more reliable abundances deduced from optical recombination lines or far-IR forbidden lines (which unfortunately are not available for the BCDs). Consequently, the abundances differences between the two gas phases of IZw 36 could be even larger than those given in Fig. 4.

At the present time, the overall picture remains unclear (see also Lecavelier et al. 2004; Aloisi et al. 2003). To assess which of the effects (unprocessed neutral gas, depletion on dust grains, metal enrichment) are dominant, it is necessary to investigate the neutral gas of more gas-rich star-forming galaxies. Furthermore, with a larger sample, we can reasonnably expect to find several good oxygen determinations.

Acknowledgements. This work is based on data obtained for the French Guaranteed Time and the PI Team Guaranteed Time by the NASA-CNES-CSA FUSE mission operated by the Johns Hopkins University. French participants are supported by CNES. This work has been done using the profile fitting procedure Owens. $f$ developed by M. Lemoine and the FUSE French Team. We are very grateful to Martial André for providing useful comments.

\section{References}

Aloisi, A., Savaglio, S., Heckman, T. M., et al. 2003, ApJ, 595, 760

André, M. K., Oliveira, C. M., Howk, J. C., et al. 2003, ApJ, 591, 1000

Deharveng, J.-M., Albrecht, R., Barbieri, C., et al. 1994, A\&A, 288, 413

Hébrard, G., Lemoine, M., Vidal-Madjar, A., et al. 2002, ApJS, 140, 103

Heiles, C. 1975, A\&AS, 20, 37

Izotov, Y. I., Thuan, T. X., \& Lipovetsky, V. A. 1997, ApJS, 108, 1

Izotov, Y. I., \& Thuan, T. X. 1999, ApJ, 511, 639

Jenkins, E. B., Oegerle, W. R., Gry, C., et al. 2000, ApJ, 538, L81

Kunth, D., \& Sargent, W. L. W. 1986, ApJ, 300, 496

Kunth, D., Lequeux, J., Sargent, W. L. W., \& Viallefond, F. 1994, A\&A, 282, 709

Lebouteiller, V. 2003, in preparation

Lecavelier des Etangs, A., Désert, J.-M., Kunth, D., et al. 2004, A\&A, 413, 131

Legrand, F. 2000, A\&A, 354, 504

Lodders, K. 2003, ApJ, 591, 1220

Moos, H. W., Cash, W. C., Cowie, L. L., et al. 2000, ApJ, 538, L1

Pettini, M., \& Lipman, K. 1995, A\&A, 297, L63

Savage, B. D., \& Sembach, K. R. 1996, ARA\&A, 34, 279

Schulte-Ladbeck, R. E., Hopp, U., Greggio, L., Crone, M. M., \& Drozdovsky, I. O. 2001, AJ, 121, 3007

Searle, L., \& Sargent, W. L. W. 1972, ApJ, 173, 25

Sofia, U. J., \& Jenkins, E. B. 1998, ApJ, 499, 951

Stasinska, G. 1990, A\&AS, 83, 501

Tenorio-Tagle, G. 1996, AJ, 111, 1641

Thuan, T. X., Lecavelier des Etangs, A., \& Izotov, Y. I. 2002, ApJ, 565,941

Thuan, T. X., \& Lecavelier des Etangs, A. 2003, in preparation

Tsamis, Y. G., Barlow, M. J., Liu, X.-W., Danziger, I. J., \& Storey, P. J. 2003, MNRAS, 338, 687

Viallefond, F., \& Thuan, T. X. 1983, ApJ, 269, 444 (V83)

Vidal-Madjar, A., Kunth, D., Lecavelier des Etangs, A., et al. 2000, ApJ, 538, L77 